\title{
Revisión sistemática: células madre derivadas del tejido adiposo, utilidad en lipoatrofia facial
}

\author{
Systematic review: stem cells derived from adipose tissue, \\ utility in facial lipoatrophy
}

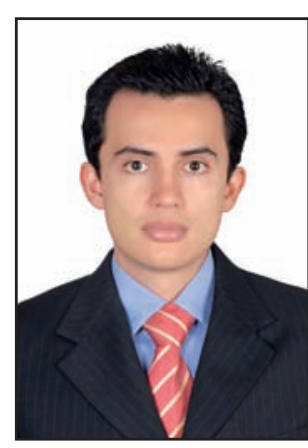

Cruz-Sánchez, M.D.

Cruz-Sánchez, M.D.*, Gómez-Díaz. O.**

Resumen

El objetivo de la presente revisión sistemática es determinar si los lipoinjertos enriquecidos con células madre derivadas del tejido adiposo son superiores a los injertos de tejido graso convencionales en el tratamiento de la lipoatrofia facial.

Realizamos una búsqueda sistemática de publicaciones en las bases de datos: MEDLINE, SCOPUS, OVID SP, SCIELO y EMBASE y posteriormente, una valoración basada en criterios actuales de medicina basada en la evidencia de las publicaciones encontradas por dos revisores independientes; las discrepancias en el análisis se resolvieron por acuerdo. Los criterios de inclusión fueron: ensayos clínicos aleatorizados, series clínicas y reportes de casos en humanos, excluyendo los ensayos en animales.

Encontramos 6 publicaciones que cumplían con los criterios previamente mencionados. Entre éstas se incluyeron 2 ensayos clínicos aleatorizados controlados que recogían la superioridad de los lipoinjertos enriquecidos con células madre derivadas del tejido adiposo en el tratamiento de la lipoatrofia facial en términos de resistencia a la reabsorción (preservación del volumen) y menor necesidad de nuevos lipoinjertos, en comparación con los lipoinjertos convencionales.

Concluimos diciendo que existen publicaciones con buenos niveles de evidencia que sugieren que los lipoinjertos enriquecidos con células madre derivadas del tejido adiposo son superiores a los lipoinjertos convencionales en el tratamiento de la lipoatrofia facial. No obstante, son estudios de reciente publicación y aún es necesario tener seguimientos a más largo plazo y con mayor número de pacientes para mejorar los ya buenos niveles de evidencia disponibles.

\begin{tabular}{|l} 
Palabras clave $\begin{array}{l}\text { Atrofia facial, Lipoatrofia facial, Hemiatrofia } \\
\text { facial, Lipoinjertos, Células madre, } \\
\text { Lipoinjertos enriquecidos, Evidencia científica. }\end{array}$ \\
Nivel de evidencia científica II
\end{tabular}

Abstract

The objective of this systematic review is to determine if the lipografts enriched with stem cells derived from adipose tissue are superior to the conventional fatty tissue grafts in the treatment of facial lipoatrophy.

A systematic search was performed for publications in the databases: MEDLINE, SCOPUS, OVID SP, SCIELO and EMBASE. An evaluation was subsequently conducted based on current criteria of evidence-based medicine of the publications found by two independent reviewers and discrepancies in the analysis were resolved by agreement. The inclusion criteria were: randomized clinical trials, clinical series and case reports in humans; the reports in animals were excluded.

We found 6 publications that meet the criteria previously mentioned. Between these, 2 randomized controlled clinical trials reported the superiority of the lipografts enriched with stem cells derived from adipose tissue in the treatment of facial lipoatrophy in terms of resistance to the reabsorption (volume preservation) and less need for new lipografts when compared with the conventional lipografts.

We conclude that there are publications with good levels of evidence to suggest that the lipografts enriched with stem cells derived from adipose tissue are better than the conventional lipografts in the treatment of facial lipoatrophy. However, these are studies of recent publication and it is still necessary to have long-term follow-ups and with a greater number of patients to improve the already good levels of evidence available.
Key words Facial atrophy, Facial lipoatrophy, Facial hemiatrophy, Lipografts, Stem cells, Enriched lipografts, Scientific evidence.

Level of evidence II

\footnotetext{
* Médico Residente.

** Cirujano plástico, Jefe del Servicio.
} 


\section{Introducción}

La atrofia facial bilateral o la hemiatrofia facial son entidades que pueden tener múltiples etiologías; desde patologías autoinmunes como el lupus (1) y la esclerodermia (2), pasando por síndromes que no tienen aún una causa definida como el Parry Romberg (3) y el Barraquer Simons (4), para terminar en atrofias faciales relacionadas con patologías infecciosas como la producida por el virus de la inmunodeficiencia humana (5).

Durante años, se han empleado los lipoinjertos convencionales para el tratamiento de estas patologías; no obstante, inconvenientes como la reabsorción del injerto (pérdida de volumen) y la necesidad de múltiples procedimientos para lograr resultados satisfactorios, han impulsado la búsqueda de nuevas herramientas terapéuticas.

Las células madre derivadas del tejido adiposo (ADSC) son células madre mesenquimales multipotentes obtenidas del tejido adiposo que se pueden diferenciar en múltiples linajes (6). Estos incluyen los siguientes tipos celulares: osteoblastos, células endoteliales, adipocitos, condrocitos, células hematopoyéticas, cardiomiocitos, e incluso algunos autores refieren que hay diferenciación hacia células pancreáticas y células neuronales (7). Identificadas por Zuk en el 2001 (8), estas células pueden dar origen a múltiples tipos de tejido, hecho del que deriva su potencial utilidad clínica.

Se desconoce el origen de estas células, aunque se postula que pueden ser pericitos que rodean los vasos sanguíneos del tejido adiposo; esto podría explicar su capacidad para diferenciarse a células endoteliales. Otros autores proponen que podrían ser una subpoblación de fibroblastos que residen en el tejido adiposo (9). Generalmente, las células que se utilizan en los estudios son células autólogas, lo que prácticamente elimina el riesgo de rechazo inmunológico.

Una vez aplicadas en el tejido receptor, las ADSC ejercen los siguientes efectos biológicos (6):

- Angiogénesis y aumento de la síntesis de colágeno a través de los siguientes factores de crecimiento: factor de crecimiento endotelial vascular, factor de crecimiento transformante beta y factor de crecimiento derivado de plaquetas. Por este mecanismo se explicaría su potencial utilidad para mantener viable y disminuir la reabsorción de un lipoinjerto enriquecido con ADSC.

- Inmunomodulación: estas células no expresan el HLA-DR (antígeno leucocitario humano-DR), suprimen la proliferación de linfocitos y potencian las citoquinas antinflamatorias.

El proceso de extracción de las ADSC y enriquecimiento de los lipoinjertos se realiza a través de varios métodos $(6,7)$ descritos con detalle en los artículos que referenciamos. De forma resumida podemos decir que en la actualidad, la mayoría de publicaciones con aplicación en Cirugía Plástica, realizan este proceso siguiendo los pasos que enumeraremos a continuación o variaciones de los mismos:

- Calcular el volumen del defecto a rellenar.

- Seleccionar el área donante del lipoinjerto: el sitio donante que más se utiliza en los ensayos clínicos es la grasa abdominal puesto que se cree que produce un mayor rendimiento de ADSC (10). Sin embargo, se pueden utilizar otras áreas como los flancos o los muslos.

- Realizar liposucción de un volumen que, en la mayoría de las investigaciones publicadas, es mayor al del defecto a rellenar.

- Agregar al lipoaspirado colagenasa durante 30 a 60 minutos, en condiciones de agitación constante para que la enzima digiera el lipoaspirado y después de centrifugar esta mezcla, obtener un precipitado denominado fracción estromal vascular (SVF) que contiene: eritrocitos, fibroblastos, pericitos, células endoteliales y ADSC. El contenido de células madre puede variar, encontrándo hasta un $22 \%$ de mayor rendimiento en los lipoaspirados obtenidos del tronco con respecto a los obtenidos de las extremidades (11).

- Posteriormente se lleva la SVF a cultivo sobre plástico; de esta mezcla de células, solo las ADSC tendrán la propiedad de adherirse al plástico, hecho que permite su aislamiento.

- Todo este procedimiento debe efectuarse en condiciones de extrema esterilidad.

Es importante mencionar que hay investigaciones clínicas que enriquecen el lipoinjerto con la SVF y lo aplican directamente al paciente en la misma intervención quirúrgica. Si bien este lipoinjerto tendría una mayor proporción de ADSC, también tendría otras células.

Otros autores llevan la SVF o una biopsia de tejido adiposo a cultivo y posteriormente identifican marcadores de membrana específicos de ADSC con el objetivo de ser más selectivos en la clasificación de las células, si bien este procedimiento suele tardar varios días e implica un segundo procedimiento quirúrgico.

Varios de los ensayos referenciados en el presente estudio utilizan las ADSC una vez obtenidas del paciente o en un corto plazo. No obstante, estas células se pueden almacenar en bancos durante años mediante criopreservación, de manera que en el momento de ser requeridas por el paciente se descongelan pudiéndose recuperar alrededor del $80 \%$ de las mismas (12).

El objetivo de la revisión sistemática que presentamos es determinar si los lipoinjertos enriquecidos con ADSC son superiores a los injertos de tejido graso convencionales en el tratamiento de la lipoatrofia facial. 


\section{Material y Método}

Llevamos a cabo una búsqueda sistemática en las bases de datos: MEDLINE, SCOPUS, OVID SP, SCIELO y EMBASE, utilizando los términos de búsqueda ilustrados en la Tabla I. Posteriormente, hicimos una selección de los artículos, sin restricción de idioma, que cumplían con los criterios de inclusión que nombraremos a continuación y fueron revisados por 2 revisores independientes que resolvieron las discrepancias en el análisis mediante acuerdo.

Los criterios de inclusión utilizados fueron:

- Ensayos clínicos aleatorizados, series clínicas y reportes de casos en humanos.

Como criterios de exclusión:

- Estudios en animales e in vitro

Dada la heterogeneidad en la forma de presentar los resultados de los estudios seleccionados, realizamos una revisión sistemática narrativa y tomamos en cuenta la metodología de clasificación de la evidencia médica de la Universidad de Oxford (Tabla II) para clasificar las publicaciones seleccionadas.

\section{RESULTADOS}

De las publicaciones encontradas solamente 6 trataban el abordaje de la lipoatrofia facial con ADSC y a la vez cumplían con los criterios de inclusión previamente mencionados (Tabla III). A continuación, describimos los hallazgos más importantes de estos 6 artículos.

Citamos en primer lugar un ensayo clínico aleatorizado realizado por Sterodimas y col. en Brasil (13), en el que se reclutó un grupo de 20 pacientes con lipoatrofia facial con los siguientes diagnósticos: síndrome de Barraquer-Simons (5 pacientes), enfermedad de Parry Romberg (3 pacientes), atrofia facial (5 pacientes), defectos postraumáticos (6 pacientes) y lupus eritematoso sistémico (1 paciente), todos distribuídos de forma aleatoria en 2 grupos de 10 pacientes cada uno. Un grupo recibió un injerto autólogo convencional de tejido graso obtenido por liposucción del abdomen, y el otro grupo recibió el mismo injerto autólogo de igual origen pero enriquecido con ADSC. La satisfacción global de los pacientes se evaluó a los 6, 12 y 18 meses del procedimiento quirúrgico inicial, empleando una escala previamente validada por el autor: $1=$ pobre, $2=$ aceptable, 3 = bueno, $4=$ muy bueno, $5=$ excelente .

Tabla I. Términos de búsqueda bibliográfica empleados para el estudio

\begin{tabular}{|c|c|}
\hline Tratamiento de la lipoatrofia facial & Términos de búsqueda \\
\hline & $\begin{array}{l}\text { "Facial Hemiatrophy"[Mesh] OR Romberg Facial Hemiatrophy [text word] OR } \\
\text { Romberg Disease [text word] OR Parry-Romberg Disease [text word] OR Parry } \\
\text { Romberg Disease [text word] OR Parry-Romberg Syndrome [text word] OR } \\
\text { Parry Romberg Syndrome [text word] OR Progressive Facial Hemiatrophy [text } \\
\text { word] OR Progressive Hemifacial Atrophy [text word] OR Hemifacial Atrophy, } \\
\text { Progressive [text word] OR Progressive Hemifacial Atrophies [text word] OR } \\
\text { Romberg's Disease [text word] OR Facial Hemiatrophies [text word] OR facial } \\
\text { atrophy [text word] OR facial lipoatrophy[text word] OR HIV associated facial } \\
\text { lipoatrophy [text word] AND Adipose derived stem cells [text word] OR enriched } \\
\text { lipografts [text word] OR Adipose-Derived Stem Cell-Enriched lipografts[text } \\
\text { word] }\end{array}$ \\
\hline
\end{tabular}

Tabla II. Clasificación de la evidencia médica de la Universidad de Oxford

\begin{tabular}{|c|l|}
\hline Nivel de Evidencia & \multicolumn{1}{c|}{ Tipo de Estudio } \\
\hline $1 \mathrm{~A}$ & Revisión sistemática de ensayos clínicos controlados (homogéneos entre si) \\
\hline 1B & Ensayos clínicos controlados (con intervalo de confianza estrecho) \\
\hline $2 \mathrm{~A}$ & Revisión sistemática de estudios de cohorte (homogéneos entre si) \\
\hline $2 \mathrm{~B}$ & Estudio individual de cohortes/ ECA individual de baja calidad \\
\hline $3 \mathrm{~A}$ & Revisión sistemática de casos y controles (homogéneos entre si) \\
\hline $3 \mathrm{~B}$ & Estudio individual de casos y controles \\
\hline 4 & Series de casos, estudios de cohorte / casos y controles de baja calidad \\
\hline 5 & Opiniones de expertos basados en revisión no sistemática de resultados o esquemas fisioplatológicos \\
\hline
\end{tabular}


Tabla III. Estudios acerca del uso de lipoinjertos enriquecidos con células madre derivadas del tejido adiposo en pacientes con lipoatrofia facial

\begin{tabular}{|l|l|c|c|}
\hline \multicolumn{1}{|c|}{ Autor principal } & \multicolumn{1}{|c|}{ Publicación } & $\begin{array}{c}\text { Número } \\
\text { de pacientes }\end{array}$ & $\begin{array}{c}\text { Nivel } \\
\text { de evidencia }\end{array}$ \\
\hline Sterodimas & $\begin{array}{l}\text { Autologous Fat Transplantation Versus Adipose-Derived } \\
\text { Stem Cell-Enriched Lipografts: A Study. Aesth. Surg. J. } \\
\text { 2011;31(6) :682-693. }\end{array}$ & 20 & $2 \mathrm{~b}$ \\
\hline Koh & $\begin{array}{l}\text { Clinical Application of Human Adipose Tissue Derived Me- } \\
\text { senchymal Stem Cells in Progressive Hemifacial Atrophy } \\
\text { (Parry-Romberg Disease) With Microfat Grafting Techniques } \\
\text { Using 3-Dimensional Computed Tomography and 3-Dimen- } \\
\text { sional Camera. Ann Plast Surg. 2012;69(3): 331-337. }\end{array}$ & 10 & $2 \mathrm{~b}$ \\
\hline Yoshimura & $\begin{array}{l}\text { Cell-assisted lipotransfer for facial lipoatrophy: Efficacy of } \\
\text { clinical use of adipose derived stem cells. Dermatol Surg. } \\
\text { 2008;34: 1178-1185. }\end{array}$ & 6 & 4 \\
\hline Arana & $\begin{array}{l}\text { Lipoinfiltrado enriquecido con células madre en población } \\
\text { pediátrica con síndrome de Parry-Romberg. Actualización. } \\
\text { Cir.plást. iberolatinoam.2013, 39 (Supl. 1): S99-S106. }\end{array}$ & 6 & 4 \\
\hline Castro & $\begin{array}{l}\text { Cell-assisted lipotransfer for the treatment of parry-romberg } \\
\text { syndrome. Arch Plast Surg. 2012;39(6):659-662. }\end{array}$ & 1 & 4 \\
\hline Mehmet & $\begin{array}{l}\text { Treatment of “En Coup de Sabre” Deformity With Adipose- } \\
\text { Derived Regenerative Cell-Enriched Fat Graft. J Craniofac } \\
\text { Surg. 2012;23(2):e103-105. }\end{array}$ & 1 & 4 \\
\hline
\end{tabular}

En el grupo de injerto de tejido adiposo autólogo convencional, 3 pacientes lograron resultados estéticamente aceptables después del primer tratamiento; los restantes 7 pacientes requirieron sesiones de injerto adicionales para obtener resultados favorables. En el grupo de injerto graso autólogo enriquecido con ADSC, todos los pacientes requirieron solamente 1 sesión de injerto para lograr buenos resultados.

El análisis de satisfacción de los pacientes a los primeros 6 meses, demostró claramente mejores resultados en el grupo de injerto enriquecido con células madre. No obstante, en el seguimiento a los 18 meses no hubo diferencia estadísticamente significativa. Sin embargo, al analizar el número de sesiones de injerto graso, notoriamente se favorece al grupo de injertos enriquecidos con células madre ya que solamente fue necesario 1 procedimiento quirúrgico en todos los pacientes, mientras que en el grupo de injerto autólogo convencional fueron necesarias 2 intervenciones en 3 pacientes y 3 intervenciones en 4 pacientes.

En segundo lugar citamos otro ensayo clínico aleatorizado publicado por Kyung Suk Koh (14) en Corea del Sur. Este autor toma 10 pacientes con diagnóstico de enfermedad de Parry-Romberg y los asigna de manera aleatorio a 2 grupos, cada uno con 5 pacientes. El grupo control recibió lipoinjerto de grasa abdominal convencional en el día 0 y un nuevo injerto de tejido graso convencional en el día 14; el grupo experimental recibió injerto de tejido graso abdominal convencional en el día 0 y un nuevo injerto de tejido graso abdominal enrique- cido con ADSC aisladas en cultivo, a una concentración de $1 \times 10^{7}$ ADSC el día 14 .

El volumen del defecto se midió en el preoperatorio y en el postoperatorio con una cámara de tercera dimensión y tomografía axial computarizada (TAC) también con reconstrucción en tercera dimensión. El contorno facial se evaluó a los 1, 6, 12 y 18 meses tras los injertos. Adicionalmente, se evaluó la satisfacción del paciente con una puntuación de 0 a 5 , donde 5 es la mejor calificación. Este estudio encuentra que las puntuaciones de satisfacción de los pacientes en el grupo experimental eran mayores (media de 4,5) en comparación con las puntuaciones en el grupo control (media: 3,1$)$. La reabsorción del injerto en el grupo experimental fue del $20,59 \%$, mientras que en el grupo control fue del $46,81 \%$. Antes de la cirugía, la diferencia media entre la hemicara ipsilateral al defecto y la contralateral a este en términos de volumen en los pacientes del grupo experimental fue $21,71 \mathrm{ml}$, bajando a $4,47 \mathrm{ml}$ después de la cirugía. La diferencia media preoperatoria en volumen entre las dos hemicaras en los pacientes del grupo control fue de $8,32 \mathrm{ml}$, disminuyendo a $3,89 \mathrm{ml}$ después de la cirugía. Las diferencias de volumen pre y postoperatorio entre los grupos fueron estadísticamente significativas $(\mathrm{p}=0,002)$.

En tercera instancia nos referimos al trabajo realizado por Arana y col. en España (15) que analiza los resultados de 6 adolescentes con síndrome de Parry-Romberg a los que trata con lipoinjerto enriquecido con ADSC. A 5 de los 6 pacientes de la serie se les realiza un segundo 
lipoinjerto enriquecido a los 12 meses, posiblemente debido a la severidad de la lipoatrofia que padecían. Adicionalmente, en 3 de los pacientes se llevaron a cabo procedimientos complementarios como: rinoseptoplastia, colgajos miomucosos labiales de avance V-Y y una otoplastia unilateral.

En todos los pacientes se realizaron estudios de imagen pre y postoperatorios a los 6 y a los 12 meses de la cirugía mediante resonancia nuclear magnética (RNM), realizando medidas comparativas entre el grosor de los tejidos blandos y la simetría facial pre y postoperatoria. Finalmente, el estudio se complementa con la valoración del grado de satisfacción de los pacientes, de su familia y del equipo médico.

Después de un periodo de seguimiento medio de 25 meses, la autora concluye que consiguió resultados satisfactorios en variables como estabilidad del lipoinjerto, mejoría en la textura, pigmentación de la piel suprayacente al lipoinjerto y complacencia global del paciente y la familia.

Otro estudio prospectivo, éste no aleatorizado, realizado por Yoshimura en Japón (16), incluye 6 pacientes con lipoatrofia facial, 5 de ellos con diagnóstico de lupus eritematoso profundo y 1 con enfermedad de Parry Romberg. Divide a los participantes en 2 grupos, cada uno de ellos con 3 pacientes. Al primer grupo le aplica un lipoinjerto convencional obtenido de la grasa subcutánea del abdomen, y al segundo grupo un lipoinjerto enriquecido con ADSC también obtenido de la grasa subcutánea del abdomen.

El autor toma fotografías de cada paciente antes y después del procedimiento quirúrgico y 4 cirujanos plásticos certificados, que desconocen el tipo de intervención realizada en los pacientes, evalúan el porcentaje de mejoría volumétrica de los defectos faciales tratados. Clasifica la media de la mejoría volumétrica de cada paciente en 4 categorías: excelente ( $80 \%$ de mejoría o más), buena (del $60 \%$ a menos del $80 \%$ de mejoría), aceptable (del $40 \%$ a menos del $60 \%$ de mejoría) y pobre (menos del $40 \%$ de mejoría). Luego realiza un seguimiento de entre 9-13 meses a cada uno de los participantes en el estudio. El grupo que recibió el lipoinjerto con células madre tuvo una mejor puntuación clínica respecto a mejoría volumétrica del defecto en el postoperatorio que el grupo que solo recibió el injerto graso convencional; no obstante la diferencia no fue estadísticamente significativa $(p=0,11)$. En el seguimiento posterior a 2 meses, después de la contracción volumétrica inicial esperada en ambos grupos, observa como el grupo con injerto convencional continua contrayendo el volumen del lipoinjerto mientras que el grupo de ADSC muestra persistencia del volumen.

Otro trabajo en base a 1 caso publicado en México, con enfermedad de Parry-Romberg al que se le aplicó lipoinjerto enriquecido con ADSC, muestra permanencia del lipoinjerto a los 12 meses de seguimiento (17).

Finalmente, otro caso publicado en Turquía (18) de una paciente de sexo femenino de 19 años con esclerodermia lineal, deformidad en coup de sabre tratada con lipoinjerto enriquecido con ADSC y seguida durante 12 meses, muestra persistencia del volumen del lipoinjerto y resultados cosméticos satisfactorios para la paciente.

\section{Discusión}

Los cirujanos plásticos, que históricamente hemos manejado las reservas de grasa subcutánea al realizar liposucciones y procedimientos para modelar el contorno corporal (9), estamos llamados también a ser pioneros en el uso de los lipoinjertos enriquecidos con ADSC.

Aunque los injertos de tejido adiposo se han venido utilizando durante décadas, la técnica se consideraba en conjunto poco útil dado que el injerto tendía a la reabsorción con el tiempo (19). El descubrimiento de las ADSC en la grasa subcutánea ha despertado un nuevo interés en la materia en base a la teórica menor reabsorción que se produciría con la concentración de estas células en el injerto; no obstante, probar esta hipótesis en el terreno clínico de un problema concreto como es la lipoatrofia facial a la luz de la evidencia médica, es una tarea para la cual no encontramos antecedentes en la literatura científica.

En la actualidad existen múltiples estudios en todo el mundo que utilizan los lipoinjertos enriquecidos con ADSC en varios problemas clínicos del campo de la Cirugía Plástica $(6,7)$. Sin embargo, son pocas las aplicaciones que cuentan con ensayos aleatorizados controlados que respalden su utilidad clínica, y todavía más escasas o ausentes las revisiones sistemáticas basadas en la evidencia que evalúan la literatura científica disponible sobre este tópico.

La lipoatrofia facial de múltiples etiologías fue uno de los primeros problemas en ser abordados por uno de los pioneros en el uso de los lipoinjertos enriquecidos con ADSC como es Yoshimura (16).

Del análisis crítico y la revisión basada en la evidencia de las 6 publicaciones que analizamos en el presente estudio y que citamos en la sección de resultados, podemos decir que todas ellas incluyeron al menos variables como: puntuación de satisfacción de los pacientes con el aspecto de su rostro, tasa de reabsorción (pérdida de volumen) del lipoinjerto y necesidad de nuevos procedimientos quirúrgicos.

Empezaremos mencionando el pionero estudio prospectivo de Yoshimura (16) en el 2008, en el que el autor diseña una pequeña cohorte de 3 pacientes con lipoatrofia facial a quienes aplica lipoinjertos enriquecidos con ADSC, observando una menor reabsorción del lipoinjerto en comparación con otro grupo de 3 pacientes que recibieron lipoinjerto convencional. Pese a que se trata de un trabajo innovador, el modelo metodológico de este estudio, cuando se evalúa con la escala de Oxford (20) de evidencia médica, es un estudio nivel 4 que no permite sacar 
conclusiones a favor o en contra de los lipoinjertos enriquecidos con ADSC en comparación con los lipoinjertos convencionales en el tratamiento de la lipoatrofia facial.

En el año 2011, Sterodimas (13) publica el primer ensayo aleatorizado con nivel de evidencia $2 \mathrm{~B}$ y que incluye 20 pacientes con lipoatrofia facial de múltiples etiologías divididos en 2 grupos, cada uno de 10 pacientes, para recibir lipoinjerto enriquecido o convencional, y realiza un seguimiento a 18 meses demostrando mejores puntuaciones de satisfacción por parte de los pacientes y menor necesidad de reintervenciones en el grupo que recibió lipoinjertos enriquecidos con ADSC.

Arana (15) publica en el año 2013 una serie clínica retrospectiva, con nivel de evidencia 4, que utiliza lipoinjertos enriquecidos con ADSC en 6 adolescentes con síndrome de Parry Romberg que presentan, en un porcentaje importante, lipoatrofia facial severa. Posiblemente esta severidad en la afectación sea la causa por la cual 5 de ellos requirieron un nuevo lipoinjerto durante el seguimiento. No obstante, de esta publicación debemos resaltar la satisfacción con los resultados por parte de los pacientes y de sus familias, así como la estabilidad de los lipoinjertos evaluada mediante seguimiento clínico y por imagen, lo cual nos indica que el beneficio de utilizar ADSC también se produciría en adolescentes.

Encontramos también 2 publicaciones de casos clínicos $(17,18)$, ambos con nivel de evidencia 4 , que simplemente ilustran el uso de ADSC en patologías como la esclerodermia lineal (deformidad en cup de sabre) y en latitudes tan diversas como México y Turquía; no obstante no permiten establecer conclusiones por tratarse de casos aislados.

Finalmente, el recientemente publicado ensayo aleatorizado de Kyung Suk Koh (14), con nivel de evidencia $2 \mathrm{~B}$, incluye 10 pacientes a los que distribuye en 2 grupos, cada uno de 5 pacientes, para recibir lipoinjerto enriquecido o convencional y sigue durante 18 meses. Introduce además de las variables subjetivas de satisfacción del paciente tenidas en cuenta por Sterodimas, mediciones objetivas del comportamiento del lipoinjerto en el tiempo con respecto al porcentaje de reabsorción evaluado mediante TAC tridimensional y cámara fotográfica que realiza también reconstrucción tridimensional. Corrobora de esta forma los hallazgos de Sterodimas y Yoshimura respecto a una mayor satisfacción del paciente, menor reabsorción del lipoinjerto en el tiempo y menor necesidad de reintervención, con el uso de ADSC.

\section{Conclusiones}

Concluimos esta revisión sistemática de lipoatrofia facial mencionando que hemos podido encontrar en la literatura al respecto, 2 ensayos aleatorizados con nivel de evidencia $2 \mathrm{~B}$ que demuestran la superioridad de los li- poinjertos enriquecidos con células madre derivadas del tejido adiposo frente a los lipoinjertos convencionales en el tratamiento de la atrofia facial. Esta superioridad se expresa en términos de mejores puntuaciones de satisfacción por parte de los pacientes con respecto al aspecto de su rostro, una menor tasa de reabsorción (pérdida de volumen) del lipoinjerto en el tiempo y una menor necesidad de nuevos procedimientos quirúrgicos para lograr los resultados deseados.

Agradecimiento

Agradecemos la valiosa colaboración del profesor Juan Manuel Reyes, de la División de Epidemiología de la Facultad de Medicina de la Universidad Nacional de Colombia.

\section{Dirección del autor}

\section{Dr. Mario Didi Cruz Sánchez}

Carrera 14 \# 1-05 Bloque 7 Apartamento 508

Bogotá, Colombia

e-mail: mdcruzs@unal.edu.co

\section{Bibliografía}

1. Arai S. and Katsuoka K.: Clinical entity of Lupus erythematosus panniculitis/lupus erythematosus profundus. Autoimmunity Reviews. 2009 ; 8(6): 449-452.

2. Torok KS.: Pediatric scleroderma: systemic or localized forms. Pediatr Clin North Am. 2012;59(2):381-405.

3. El-Kehdy J, Abbas O, Rubeiz N.: A review of ParryRomberg syndrome. J Am Acad Dermatol. 2012; 67(4):769-784.

4. Mansouri Hattab N, Lahmiti S, Aimadeddine S et al.: Barraquer-Simons syndrome. Rev Stomatol Chir Maxillofac. $2011 ; 112(3): 172-173$.

5. Guaraldi G, Fontdevila J, Christensen L et al.: Surgical correction of HIV-associated facial lipoatrophy. AIDS. $2011 ; 25: 1-12$.

6. Gir P., Oni G., Spencer A y col.: Human Adipose Stem Cells: Current Clinical Applications. Plast. Reconstr. Surg. 2012 ;129: 1277-1290.

7. Lasso, JM, Pérez Cano, R.: Estado actual de las terapias con células madre derivadas de tejido adiposo en el ámbito de la Cirugía Plástica. Cir.plást. iberolatinoam 2010; 36(3): 215-221.

8. Zuk PA, Zhu M, Mizuno H: Multi-lineage cells from human adipose tissue: implication for cell-based therapies. Tissue Eng 2001; 7: 211.

9. Neligan P, Gurtner G.: Stem cells and regenerative medicine. En: Plastic Surgery. Vol I. Ed: Elsevier. 2013. Pp: 212-239.

10. Jurgens WJ, Oedayrajsingh-Varma MJ, Helder MN.: Effect of tissue-harvesting site on yield of stem cells derived from adipose tissue: implications for cell-based therapies. Cell Tissue Res 2008;332:415-426.

11. Almeida, K. A. et al.: Fracción vascular estromal de tejido adiposo: cómo obtener células madre y su rendimiento de acuerdo a la topografía de las áreas donantes: estudio preliminar. Cir. plást. iberolatinoam., 2008, 34 (1): .71-77. 
12. Planas Ribo, J. and Coronel Gagliardi, R.: Obtención y criopreservación de células madre del tejido graso mediante liposucción. Cir. plást. iberolatinoam., 2011, 37 (4): 319-324.

13. Sterodimas A, De Faria J, Nicaretta B y col.: Autologous Fat Transplantation Versus Adipose-Derived Stem Cell-Enriched Lipografts: A Study. Aesth. Surg. J. 2011; 31(6) :682-693.

14. Koh KS, Oh TS, Kim H y col.: Clinical Application of Human Adipose Tissue Derived Mesenchymal Stem Cells in Progressive Hemifacial Atrophy (Parry-Romberg Disease) With Microfat Grafting Techniques Using 3-Dimensional Computed Tomography and 3-Dimensional Camera. Ann Plast Surg. 2012;69(3): 331-337.

15. Arana E. Pérez M, Barret J.P.: Lipoinfiltrado enriquecido con células madre en población pediátrica con síndrome de Parry-Romberg. Actualización. Cir.plást. iberolatinoam. 2013, 39 (Supl. 1): S99-S106.
16. Yoshimura K, Sato K, Aoi N.: Cell-assisted lipotransfer for facial lipoatrophy: Efficacy of clinical use of adipose derived stem cells. Dermatol Surg. 2008;34:1178-1185.

17. Castro-Govea Y, De La Garza-Pineda O, Lara-Arias J y col.: Cell-assisted lipotransfer for the treatment of parry-romberg syndrome. Arch Plast Surg. 2012; 39(6):659-662

18. Mehmet Veli Karaaltin, Ali Cem Akpinar, Semih Baghaki y col.: Treatment of "En Coup de Sabre" Deformity With Adipose-Derived Regenerative Cell-Enriched Fat Graft. J Craniofac Surg. 2012;23(2):e103-105.

19. Benito Ruiz, J.: Injertos de tejido adiposo: variables que influyen en la viabilidad del adipocito y de las células madre mesenquimales. Cir. plást. iberolatinoam., 2011, 37 (4):311-318.

20. Bob Phillips, Chris Ball, Dave Sackett y col.: Oxford Centre for Evidence-based Medicine Levels of Evidence, 2009. http://www.cebm.net/index .aspx?o=1025. 


\title{
Comentario al artículo “Revisión sistemática: células madre derivadas del tejido adiposo, utilididad en lipoatrofia facial"
}

\author{
Dra. Elena Arana \\ Especialista en Cirugía Plástica, Estética y Reparadora. Coordinadora de Cirugía Plástica Área Maternoinfantil, Hospital \\ Universitario Valle de Hebrón, Barcelona, España.
}

Primero agradecer la oportunidad que se me ofrece de comentar el estupendo trabajo de los Dres. Cruz-Sánchez y Gómez-Díaz al tratar sobre un tema que me interesa especialmente y siendo además que se me incluye entre los artículos revisados.

Resulta interesante que los autores realicen una revisión sistemática de las publicaciones a fecha de hoy en el campo de la utilización de rellenos grasos enriquecidos con células madre en el campo de la lipoatrofia facial; entre los artículos seleccionados destacan los de Sterodimas, Koh y Yoshimura, de obligado conocimiento para todo cirujano plástico que se dedique a esta parcela.

La reunión de todos ellos en un sólo texto resulta clarificadora; al detallar la sistemática de cada trabajo, nos señala el camino a seguir si queremos mejorar en nuestro trabajo y obtener unas conclusiones más certeras:

- Preferencia por los ensayos clínicos frente a las series clínicas.

- Medición objetiva de la retención del injerto graso.

- Valoración de los resultados obtenidos mediante el análisis realizado por cirujanos plásticos competentes y ajenos al tratamiento realizado, frente a encuesta de satisfacción de los pacientes y su entorno.

- Seguimiento a largo plazo de los pacientes para valorar la permanencia en el tiempo del injerto graso.

De todos los puntos detallados, a mi parecer, el más difícil de implementar es el de la medición de los resultados obteni-

\section{Respuesta al comentario de la Dra. Arana}

\section{Dr. Mario Didi Cruz Sánchez}

Agradecemos a la Dra. Arana el comentario a nuestro artículo y su valiosa participación en la revisión sistemática con un trabajo en población de adolescentes con síndrome de Parry Romberg en la que utilizó lipoinjertos enriquecidos con células madre derivadas del tejido adiposo.

La medicina basada en la evidencia es una herramienta que nos permite evaluar el impacto de nuestros tratamientos. Someter a este tipo de valoración crítica múltiples publicaciones sobre una intervención, como se hace en una revisión sistemática, nos permite aproximarnos a cómo de reales son los efectos que creemos podemos lograr con una técnica quirúrgica con un soporte biológico, como lo es el uso de lipoinjertos enriquecidos con células madre derivadas del tejido adiposo. Sin lugar a dudas, la medición objetiva de los resultados a largo plazo con estos lipoinjertos enriquecidos en pacientes con lipoatrofia facial en términos de reabsorción, resulta una tarea difícil de realizar.

Con base en los resultados obtenidos en esta revisión, planteamos una primera aproximación a este problema según lo pu- dos; si bien es cierto que las aportaciones realizadas tanto por Koh como por Fontdevila (1) nos orientaban a realizar la medición cuantitativa de los resultados mediante técnicas de imagen, podríamos añadir nuevas estrategias como las publicadas por Serna-Cuéllar y Santamaría-Solís (2-3) analizando datos emanados del estudio directo de los injertos grasos mediante biopsia y análisis estereológico, aunque este último estudio concierne a injertos grasos tratados con la técnica de Coleman y no a injertos enriquecidos con células madre.

Por ultimo felicitar a los Dres. Cruz-Sánchez y GómezDíaz por el estupendo trabajo presentado.

\section{Bibliografía}

1. Fontdevila J,Serra-Renom JM,Raigosa M et al: Assessing the long -term viability of facial fat grafts: an objective mesure using computed tomography. Aesth. Surg. J. 2008; 28(4): 380-386.

2. Serna-Cuéllar E, Santamaría-Solís L: ¿Cual es la supervivencia de los injertos de grasa en la cara? ¿Cómo cuantificarla?: Medicina basada en la evidencia. Cir. plást. ibeolatinoam. 2013, 39 (Supl. 1): S82-S89.

3. Santamaría-Solís L Serna-Cuéllar E, Ingelmo-Ingelmo I,: Aplicación de técnicas estereológicas para la cuantificación del tejido adiposo .Cir. plást. ibeolatinoam. 2013, 39 (Supl. 1): S74-S81. blicado por los estudios pioneros en este tópico y otros estudios más recientes pero igualmente valiosos. Todos plantean el valor de combinar un conjunto de herramientas como son: el juicio clínico, las técnicas de imagenología y tal vez lo más importante, la satisfacción del paciente con el resultado, como la forma más adecuada de acercarnos a una medición más real de los efectos de esta intervención.

Como vemos, estos tratamientos que se han iniciado con series clínicas y que empiezan a reportar ensayos aleatorizados, son prometedores y seguramente en un futuro cercano, tendremos un mayor número de ensayos aleatorizados controlados que permitirán mejorar el nivel de evidencia científica.

Consideramos también que esta revisión es una adecuada justificación de cara a adquirir las tecnologías necesarias para poder ofrecer a nuestros pacientes este tipo de tratamientos en los centros asistenciales donde ejercemos diariamente. 